Original Paper http://ajol.info/index.php/ijbcs http://indexmedicus.afro.who.int

\title{
Formulation de farine de fonio enrichie en ressources alimentaires locales pour l'alimentation complémentaire des jeunes enfants au Bénin
}

\author{
Nadia FANOU FOGNY ${ }^{1^{*}}$, E. M. Yann MADODE, F.T. Flora LALEYE, \\ Yrence AMOUSSOU-LOKOSSOU et A. P. Polycarpe KAYODE \\ Ecole de Nutrition Humaine et des Sciences et Technologies Alimentaires, \\ Faculté des Sciences Agronomiques, Université d'Abomey-Calavi, Bénin. \\ *Auteur correspondant ; 07 BP 830 Cotonou, Benin ; E-mail : nadia.fanou@fsa.uac.bj
}

\section{REMERCIEMENTS}

La présente recherche a bénéficié du soutien financier de la Fondation Internationale pour la Science et du Groupe COMSTECH.

\section{RESUME}

L'étude a pour objectif de développer une bouillie de complément à base de fonio pour les enfants de 6 à 23 mois. Trois formules ont été développées sur la base des bouillies de complément habituellement consommées par les enfants. Les doses et les ingrédients ont été combinés selon les recommandations. Des analyses physico-chimiques et microbiologiques, et une évaluation sensorielle ont été effectuées sur les formules développées. Les trois formules de farine contiennent $95 \%$ (Ffo1) à $97 \%$ (FMa+) de matière sèche, et des teneurs en glucides entre $66 \mathrm{~g}(\mathrm{Ffo} 2)$ et $68 \mathrm{~g}(\mathrm{FMa}+)$ sur base sèche. En comparant les valeurs nutritionnelles aux recommandations, on obtient des taux de satisfaction de 99\% (Ffo1) à plus de 100\% (Ffo2 et FMa+) pour l'énergie, et $87 \%$ (Ffo1) à 100\% (FMa+ et Ffo2) pour les protéines. Les teneurs en lipides se situent dans l'intervalle des valeurs recommandées, et les taux de fibres sont inférieurs à $5 \mathrm{~g}$, tel que recommandé. Les teneurs théoriques en fer des trois farines sont de $2,4 \mathrm{mg}$ en moyenne, et sont largement en-deçà de la recommandation. La flore totale des trois farines est inférieure à la norme. Les farines de fonio Ffo1 et Ffo2 ont présenté une viscosité plus faible et une valeur en protéines plus élevées que celles de la farine de maïs FMa+. Globalement, $46 \%$ des mères préfèrent la bouillie de fonio Ffo2. La bouillie de fonio Ffo2 satisfait aux recommandations physico-chimiques, microbiologiques et en macronutriment. Elle contribue à la diversité alimentaire minimale des enfants de 6 à 23 mois, en combinant au moins quatre groupes d'aliments sur les sept recommandés : les céréales (fonio), les légumineuses (soja), les graines (arachide), et les poissons (fretins).

(C) 2017 International Formulae Group. All rights reserved.

Mots clés : fonio (Digitaria exilis), alimentation complémentaire, ressources alimentaires locales au Bénin, enfants de 6-23 mois, formulation de farine de céréale.

\section{Developing a fonio flour enriched with local food resources for complementary feeding of young children in Benin}

\begin{abstract}
The objective of the study is to develop a fonio-based complementary flour for children aged between 6 and 23 months. Three formulae were developed on the basis of complement porridge usually consumed by children. Amounts and ingredients were combined as recommended. Physico-chemical and microbiological
\end{abstract}


analyzes, and sensory evaluation were carried out on the developed formulae. The three flour formulae contain 95\% (Ffo1) to $97 \%(\mathrm{FMa}+$ ) of dry matter, and carbohydrate contents between $66 \mathrm{~g}(\mathrm{Ffo} 2)$ and $68 \mathrm{~g}(\mathrm{FMa}+)$ on dry basis. The nutritional values of the formulated flours meet the recommendations at: 99\% (Ffo1) to 100\% (Ffo2 and FMa +) for energy and $87 \%$ (Ffo1) to $100 \%$ (FMa + and Ffo2) for proteins. Lipid contents were within the range of recommended values, and fiber levels were less than the recommended $5 \mathrm{~g}$. Theoretical iron content of the three flours was on average $2.4 \mathrm{mg}$ and well below the guideline. The total flora of the three flours was below the maximum values. The fonio flours Ffo1 and Ffo2 showed lower viscosity and higher protein values than those of FMa (maize flour). Overall, $46 \%$ of mothers prefer Ffo2 porridge, which meets physico-chemical, microbiological and macronutrient recommendations. It also contributes to meeting the minimum dietary diversity of children aged $6-23$ months by combining at least four out of the seven recommended food groups: cereals (fonio), legumes (soya), seeds (groundnuts), and fish.

(C) 2017 International Formulae Group. All rights reserved.

Keywords: fonio (Digitaria exilis), complementary feeding, local food resources in Benin, infant $6-23$ months, cereal flour formulation.

\section{INTRODUCTION}

L'alimentation au cours des 1000 premiers jours de vie de l'enfant est critique pour son développement physique et mental à long terme (IFPRI, 2014). Dès l'âge de 6 mois, le lait maternel devient qualitativement et quantitativement insuffisant pour le nourrisson dont les besoins nutritifs sont croissants (Black et al., 2017). Il s'avère donc nécessaire d'introduire dans le régime du jeune enfant, des compléments alimentaires sous forme liquide ou semi-solide pour compléter les apports du lait maternel (OMS, 2015). La période d'alimentation complémentaire représente une période à risque élevé de carence nutritionnelle, du fait de ce changement dans l'alimentation du nourrisson habitué à se nourrir exclusivement au lait maternel (Black et al., 2017). Dans de nombreux pays en développement, la malnutrition du nourrisson et du jeune enfant, la cassure de la courbe de croissance, tout comme la mortalité des enfants sont étroitement liés à des pratiques d'alimentation de complément souvent inappropriées (Black et al., 2017). Vu l'importance de la qualité de l'alimentation complémentaire pendant cette période, des recommandations ont été faites sur la base des besoins nutritionnels des enfants au cours de cette période pour l'âge d'introduction d'aliment de complément et pour le développement des formules nutritionnellement et sanitairement adéquates d'aliments infantiles (CAC, 2013).

Cependant, dans beaucoup de pays en développement, l'aliment de complément est souvent introduit plus tôt ou plus tard que la période recommandée et les aliments de complément sont souvent inadéquats du point de vue nutritionnel et sanitaire (Vaahtera et al., 2001; OMS, 2015). Au Bénin, globalement, $27 \%$ des enfants âgés de 0 - 5 mois ont reçu des aliments de complément en plus du lait maternel alors que 59\% en ont reçu dans la tranche de 6 - 9 mois (INSAE, 2013). Les aliments de complément sont souvent composés de bouillies préparées à partir de farines de céréales et/ou de tubercules, simples ou combinées avec d'autres ingrédients mais pas souvent de façon adéquate (Brou et al., 2009). Ces bouillies sont généralement de consistance épaisse et difficile à avaler pour l'enfant. Les mères sont donc contraintes d'ajouter de l'eau dans la préparation afin de rendre les bouillies plus fluides, réduisant ainsi le taux de matière sèche et par conséquent la densité énergétique des bouillies (Elenga et al., 2009 ; Kimiywe and Chege, 2015). Lorsque le volume d'aliments consommés est de faible densité énergétique, l'enfant est exposé à un déficit en ingérés énergétiques et en protéines (Arimond et al., 2008). Aussi, la présence dans ces aliments de facteurs antinutritionnels spécifiques tels que l'acide phytique limite la 
biodisponibilité des micronutriments (Zimmermann et Hurell, 2007). L'exploration des ressources alimentaires locales représente l'une des approches alimentaires durables de lutte contre les problèmes de santé publique d'origine nutritionnelle parmi les groupes vulnérables (Toledo et Burlingame, 2006). La présente recherche s'intéresse au fonio (Digitaria exilis), céréale traditionnelle la plus ancienne de l'Afrique de l'Ouest. Encore faiblement utilisée, cette céréale a le potentiel d'améliorer la nutrition, tout en contribuant à la sécurité alimentaire et à l'utilisation durable des terres (Fogny-Fanou et al., 2009; Koreissi-Dembélé et al., 2013). La présente étude contribue à la variation des sources d'énergie et de nutriments dans l'alimentation des enfants, par l'exploration du potentiel nutritionnel d'une ressource locale faiblement utilisée. Plus spécifiquement, l'étude a pour objectif de développer et d'apprécier l'acceptabilité sensorielle d'une bouillie de complément à base de fonio pour les enfants de 6 à 23 mois au Bénin.

\section{MATERIEL ET METHODES Matériel}

Les ingrédients utilisés pour la formulation des farines sont: le maïs (Zea mays) et le fonio (Digitaria exilis), l'arachide (Arachis hypogeaea), le soja (Glycine max) et les fretins séchés (Stolothrissa tanganyicae). Ils ont été achetés sur le principal marché de la ville de cotonou.

\section{Formulation des farines}

Pour formuler des farines, une enquête pilote de consommation alimentaire a été conduite dans 50 ménages au Sud-Bénin, à partir de laquelle les bouillies habituellement consommées par les enfants le matin ont été recensées chez les mères. Dans les formules développées, le maïs et le fonio (aliments de base) représentent les principales sources d'énergie. Les sources de protéines, lipides, minéraux et vitamines ont été proportionnées entre le soja (source de protéines végétales), l'arachide (source de lipides) et les poissons fretins séchés (source de protéines animales).
Le soja a été préféré au niébé pour éviter les problèmes de flatulence et de troubles gastrointestinaux causés par sa forte teneur en azote (Laurent, 2011). Une quantité de farine de maïs germé a été incorporée dans les bouillies pour augmenter leurs densités énergétiques. Le malt est source d'alpha amylases, enzymes hydrolytiques capables de prédigérer l'amidon empêchant ainsi son gonflement (Mouquet et Trêche, 2001 ; Mouquet et al., 2003). Du malt de maïs a été ainsi produit pour réduire la viscosité des bouillies lors de la préparation. La formule a été programmée dans le tableur Excel sur la base de $100 \mathrm{~g}$ de matière sèche (Trêche, 1999), en utilisant les valeurs nutritionnelles de la table de composition des aliments de l'Afrique de l'Ouest (FAO, 2012). Les doses et les ingrédients ont été combinés en se référant aux recommandations de la Commission du Codex Alimentarius (CAC, 2013). Le Tableau 1 montre la composition en énergie, macronutriments, et en fer des trois formules standardisées de farines de bouillies de complément. La farine de maïs formulée (FMa+) est obtenue à partir de taux d'incorporation de $70 \%$ de maïs, $13 \%$ de soja, $16 \%$ d'arachide et $1 \%$ de fretins. Dans les formules à base de fonio (Ffo1 et Ffo2), en faisant la substitution du maïs au fonio, le taux d'incorporation du fonio a été maintenu pour Ffo1 et modifié pour Ffo2 (63\% de fonio, $20 \%$ de soja, $16 \%$ d'arachide et $1 \%$ de petits poissons séchés).

\section{Production des farines}

Les farines ont été produites selon le procédé standard (CAC, 2013) adapté aux caractéristiques des grains utilisés :

- Nettoyage, triage, vannage des matières premières (maïs, soja, fonio et arachide)

- Lavage de toutes les matières premières : le lavage du fonio a été fait suivant une technique appropriée pour éviter d'avoir du sable dans la farine (Koreissi-Dembélé et al., 2013).

- Egouttage et séchage au soleil sur une surface propre en protégeant les matières avec du linge propre 
- Torréfaction du maïs, du soja et de l'arachide séparément dans une poêle et à feu doux

- Dépelliculage de l'arachide

- Mélange des matières premières suivant les proportions définies ;

- Mouture du mélange dans un moulin à disque préalablement nettoyé

- Refroidissement de la farine et conditionnement dans des boîtes plastiques à l'abri de l'humidité.

\section{Standardisation des recettes des bouillies}

Les recettes des farines développées ont été standardisées en procédant à trois essais de la même recette pour chaque formule de farine. D'abord, $40 \mathrm{~g}$ de farine ont été délayés dans $50 \mathrm{ml} \mathrm{d}$ 'eau. Ensuite, un volume de $250 \mathrm{ml}$ d'eau potable est porté à ébullition. La farine délayée est versée dans l'eau à ébullition, et le mélange est constamment remué, pendant 15 minutes, à compter de l'ébullition, afin de laisser bien faire cuire le soja et réduire la teneur en facteurs antinutritionnels.

Le mélange est laissé à refroidir jusqu'à environ $45^{\circ} \mathrm{C}$, puis on y incorpore la farine de malt de maïs à $15 \%$, en remuant jusqu'à la fluidité du mélange. La bouillie obtenue est remise à cuire à feu doux, pendant 3 minutes à partir de l'ébullition.

\section{Analyses physico-chimiques et composition nutritionnelle des farines}

Le taux de matière sèche, les teneurs en lipides, en protéines, cendres, en fibres brutes ont été déterminés selon la méthode AOAC (AOAC, 2005) en triple essai. Le taux de matière sèche est déterminé par dessiccation à l'étuve à $105{ }^{\circ} \mathrm{C}$ pendant $48 \mathrm{~h}$. La teneur en lipides est déterminée par la méthode d'extraction au Soxhlet à l'aide de l'éther de pétrole comme solvant d'extraction. La teneur en protéines a été déterminée par la méthode Kjedahl. Elle permet de déterminer le taux de protéines brutes à partir de la teneur en azote à l'aide du coefficient 6,25. Les cendres ont été déterminées par incinération au four à $550{ }^{\circ} \mathrm{C}$. La détermination des fibres brutes a été faite par incinération à $550{ }^{\circ} \mathrm{C}$ pendant une heure selon la méthode décrite par Osborne et Voogt (1978). La teneur en glucides (en $\%$ de la matière sèche) a été estimée par calcul différentiel (FAO/WHO, 1998). La densité énergétique des farines a été déterminée par la méthode de la bombe calorimétrique. La mesure de la viscosité des bouillies a été faite à l'aide du consistomètre de Bostwick permettant de mesurer la vitesse d'écoulement des bouillies à $45{ }^{\circ} \mathrm{C}$ par la mesure de la distance en $\mathrm{mm}$ parcourue par le front de bouillie en 30 secondes.

\section{Analyses microbiologiques}

La composition microbiologique (flore aérobie mésophile totale, coliformes totaux et fécaux, levures et moisissures) a été analysée selon les procédures standards pour la préparation des milieux de cultures et le dénombrement des germes (Kumari et Ichhpujani, 2000). La quantité de farine à utiliser lors des analyses a été prélevée juste après obtention de chaque farine. Dix grammes de chaque farine ont été prélevés, auxquels on a ajouté $90 \mathrm{~g}$ d'eau peptonée salée (EPS). Le mélange a été homogénéisé au stomacher pendant 2 minutes pour obtenir la solution mère. Le milieu physiologique (EPS) utilisé pour la dilution a été préparé en dissolvant $5 \mathrm{~g}$ de peptone et $8,5 \mathrm{~g}$ de $\mathrm{NaCl}$ dans $1000 \mathrm{ml}$ d'eau distillée. La stérilisation est faite à $121{ }^{\circ} \mathrm{C}$ pendant 15 minutes. Les dilutions successives ont été obtenues par prélèvement à partir de pipette stérilisée de 1 $\mathrm{ml}$ de la solution mère, auxquelles sont ajoutées $9 \mathrm{ml}$ d'EPS. Chaque dilution décimale est ensemencée en double sur des milieux de culture spécifiques pour la numération des germes retenus. Les germes aérobies mésophiles totaux ont été dénombrés en milieu PCA (plate count agar) stérilisé à $121^{\circ} \mathrm{C}$ après 72 heures d'incubation à $30^{\circ} \mathrm{C}$. Les levures et moisissures ont été dénombrées en milieu MEA (malt extract agar) stérilisé à $121{ }^{\circ} \mathrm{C}$ après incubation pendant 3-5 jours en aérobie à $25{ }^{\circ} \mathrm{C}$. Les coliformes totaux et fécaux ont été dénombrés en milieu VRBG (Violet Red Bile Gélose agar) après 24 - 48 
heures d'incubation à $37 \quad{ }^{\circ} \mathrm{C}$ pour les coliformes totaux et $44{ }^{\circ} \mathrm{C}$ pour les coliformes fécaux.

\section{Evaluation sensorielle des farines}

L'évaluation sensorielle a consisté en un test hédonique appliqué à un panel de dégustateurs constitué d'enfants de 6 à 12 mois et de leurs mères (Popper et Kroll, 2005). Le test a permis aux mères de donner leur appréciation et leur préférence sur les bouillies. L'appréciation sensorielle des bouillies par les mères a été évaluée selon une échelle de cinq niveaux, de «déteste beaucoup» à «aime beaucoup». Elles ont donné leur appréciation par rapport au goût, à la couleur, à la consistance de la bouillie et une appréciation globale avec le choix d'une bouillie comme préférence. Elles ont également exprimé leur désir de donner cette bouillie à leur enfant. L'appréciation des enfants a été évaluée simplement par leur expression faciale à la prise de la bouillie sur une échelle de cinq niveaux, « déteste beaucoup $» \grave{a}$ «aime beaucoup ».

\section{Analyse statistique}

Les statistiques descriptives et l'analyse de variance (ANOVA et post-hoc Bonferroni) ont été utilisées pour analyser les résultats du test d'acceptabilité, à l'aide du logiciel XL Stat 2015, avec une valeur seuil de significativité $\alpha<0,05$.

Tableau 1 : Composition théorique des formules améliorées des bouillies de complément pour 100g de matière sèche.

\begin{tabular}{|c|c|c|c|c|c|c|c|}
\hline Ingrédients & Proportion (\%) & $\begin{array}{c}\text { Energie } \\
\text { (kcal) }\end{array}$ & $\begin{array}{l}\text { Protéine } \\
\text { (g) }\end{array}$ & $\begin{array}{l}\text { Lipide } \\
\text { g) }\end{array}$ & $\begin{array}{c}\text { Glucide } \\
\text { (g) }\end{array}$ & $\begin{array}{l}\text { Fibre } \\
\text { (g) }\end{array}$ & $\begin{array}{l}\text { Fer } \\
(\mathrm{mg})\end{array}$ \\
\hline \multicolumn{8}{|c|}{ Farine à base de maïs (FMa+) } \\
\hline Maïs & 70 & 254,1 & 6,44 & 2,66 & 50,61 & 1,05 & 2,24 \\
\hline Soja & 13 & 52,65 & 4,42 & 2,34 & 3,77 & 0,611 & 0,793 \\
\hline Arachide & 16 & 99,2 & 3,712 & 8,144 & 2,96 & 0,512 & 0 \\
\hline Fretins & 1 & 3,2 & 0,44 & 0,16 & 0 & 0 & 0,085 \\
\hline Total & 100 & 409,15 & 15,012 & 13,304 & 57,34 & 2,173 & 3,118 \\
\hline \multicolumn{8}{|c|}{ Farine à base de fonio 1 (Ffo1) } \\
\hline Fonio & 70 & 242,9 & 4,83 & 0,84 & 53,27 & 1,54 & 1,26 \\
\hline Soja & 13 & 52,65 & 4,42 & 2,34 & 3,77 & 0,611 & 0,793 \\
\hline Arachide & 16 & 99,2 & 3,712 & 8,144 & 2,96 & 0,512 & 0 \\
\hline Fretins & 1 & 3,2 & 0,44 & 0,16 & 0 & 0 & 0,085 \\
\hline Total & 100 & 397,95 & 13,402 & 11,484 & 60 & 2,663 & 2,138 \\
\hline \multicolumn{8}{|c|}{ Farine à base de fonio 2 (Ffo2) } \\
\hline Fonio & 63 & 218,61 & 4,347 & 0,756 & 47,943 & 1,386 & 1,134 \\
\hline Soja & 20 & 81 & 6,8 & 3,6 & 5,8 & 0,94 & 1,22 \\
\hline Arachide & 16 & 99,2 & 3,712 & 8,144 & 2,96 & 0,512 & 0 \\
\hline Fretins & 1 & 3,2 & 0,44 & 0,16 & 0 & 0 & 0,085 \\
\hline Total & 100 & 402,01 & 15,299 & 12,66 & 56,703 & 2,838 & 2,439 \\
\hline Normes * & & 400 & 15 & $10-25$ & nd & $<5$ & 16 \\
\hline
\end{tabular}

nd: non déterminé

*Source : CAC (Commission du Codex Alimentarius), 2003. 


\section{RESULTATS}

Le Tableau 2 montre la composition nutritionnelle et les caractéristiques physicochimiques et sanitaires des farines développées à partir des formules. Les farines de fonio Ffo1 et Ffo2 ont présenté une viscosité plus faible $(6$ et $7 \mathrm{~cm}$ ) et une valeur en protéines (18 g) visiblement plus élevées que celles de la farine de maïs FMa+ (viscosité : 10 ; protéines : $15 \mathrm{~g}$ ). La bouillie de maïs est plus visqueuse que les bouillies de fonio. Les bouillies de fonio semblent de consistance plus épaisse que la bouillie de maïs avec la même quantité de malt ajoutée.

Les trois formules de farines contiennent $95 \%$ (Ffo1) à $97 \%(\mathrm{FMa}+)$ de matière sèche, et des teneurs en glucides entre $66 \mathrm{~g}$ (Ffo2) et $68 \mathrm{~g}$ $(\mathrm{FMa}+)$ sur base sèche. Les teneurs en cendres apparaissent similaires (environ $2 \mathrm{mg}$ ) aux valeurs théoriques. En comparant les valeurs nutritionnelles aux recommandations, on obtient des taux de satisfaction de 99\% (Ffo1) à plus de $100 \%(\mathrm{Ffo} 2$ et $\mathrm{FMa}+)$ pour l'énergie et $86,67 \%$ (Ffo1) à $100 \%$ (FMa+ et Ffo2) pour les protéines. Les teneurs en lipides se situent dans l'intervalle des valeurs recommandées, en étant plus proches de la limite minimale. Les taux de fibres sont $<$ à $5 \mathrm{~g}$, tel que recommandé. Les teneurs théoriques en fer des trois farines sont de $2,4 \mathrm{mg}$ en moyenne, et sont largement en-deçà de la recommandation (Tableau 2).

Les analyses microbiologiques montrent que la valeur obtenue pour la flore totale des trois farines (charges en germes aérobies et mésophiles totaux) est inférieure à la norme (Tableau 2). Il est en de même pour leurs charges en levures et moisissures. Des coliformes totaux ont été observés dans la Ffo2, mais en quantité inférieure à la norme. Les trois farines sont exemptes de coliformes fécaux.

\section{Acceptabilité des bouillies par les enfants de 6 à 23 mois et leurs mères}

Les paramètres couleur, consistance et goût ont été évalués par les mères (Tableau 3). Globalement, $46 \%$ des femmes préfèrent la bouillie Ffo2 et $36 \%$ préfèrent la bouillie Ffo1. La bouillie de maïs est la moins appréciée (18\%). Au niveau de la consistance, il y a une différence significative dans la préférence des mères $(P=0,002)$, tandis que pour les autres paramètres il n'y a pas de différence significative.

L'observation de l'expression faciale des enfants à la consommation des bouillies montre que ces derniers n'ont pas fait de différence entre les trois bouillies (Tableau 4).

Tableau 2 : Caractéristiques physico-chimiques et composition nutritionnelle des recettes des bouillies de complément pour $100 \mathrm{~g}$ de matière sèche.

\begin{tabular}{|c|c|c|c|c|}
\hline Farines $(\mathrm{M} \pm \mathrm{ET})$ & FMa+ & Ffo1 & Ffo2 & Normes * \\
\hline Matières sèches (\%) & $97,18 \pm 0,39$ & $95,11 \pm 0,27$ & $96,31 \pm 0,09$ & \\
\hline Energie (kcal/100g) & $498,72 \pm 4,31$ & $491,82 \pm 2,95$ & $495,48 \pm 22,09$ & 400 \\
\hline Protéines(g) & $15,10 \pm 0,60$ & $18,46 \pm 1,12$ & $19,12 \pm 1,47$ & 15 \\
\hline Lipides (g) & $13,42 \pm 0,46$ & $10,60 \pm 0,27$ & $12,80 \pm 0,28$ & $10-25$ \\
\hline Glucides (g) & $67,19 \pm 1,27$ & $68,08 \pm 1,21$ & $65,58 \pm 2,12$ & Nd \\
\hline Fibres (g) & $2,16 \pm 0,08$ & $1,93 \pm 0,06$ & $2,07 \pm 010$ & $<5$ \\
\hline Cendres (mg) & $1,92 \pm 0,027$ & $1,42 \pm 0,003$ & $1,69 \pm 0,022$ & \\
\hline Viscosité (cm) & $9,83 \pm 1,18$ & $6,83 \pm 0,29$ & $5,92 \pm 0,78$ & \\
\hline
\end{tabular}


Tableau 3: Appréciation comparative des bouillies par les mères d'enfants de 6 à 23 mois $(n=50)$.

\begin{tabular}{lccccc}
\hline Bouillies & Couleur & Consistance & Goût & Appréciation globale & Préférence (\%) \\
\hline FMa + & $4,440 \mathrm{a}$ & $3,260 \mathrm{~b}$ & $4,500 \mathrm{a}$ & $4,400 \mathrm{a}$ & 18 \\
\hline Ffo1 & $4,760 \mathrm{a}$ & $4,140 \mathrm{a}$ & $4,520 \mathrm{a}$ & $4,480 \mathrm{a}$ & 36 \\
\hline Ffo 2 & $4,600 \mathrm{a}$ & $4,140 \mathrm{a}$ & $4,440 \mathrm{a}$ & $4,420 \mathrm{a}$ & 46 \\
\hline P $($ ANOVA $)$ & 0,160 & 0,002 & 0,911 & 0,913 & - \\
\hline
\end{tabular}

Farine à base de maïs (FMa+); Farine à base de fonio 1 (Ffo1); Farine à base de fonio 2 (Ffo2).

Les chiffres affectés de la même lettre sur une même colonne ne sont pas significativement différents $(\mathrm{p}<0,05)$.

Tableau 4 : Appréciation comparative des bouillies par les enfants de 6 à 23 mois $(n=50)$.

\begin{tabular}{cccccc}
\hline Bouillie & Effectif & Moyenne estimée & Groupes & Bouillies comparées & P $^{*}$ \\
\hline FMa + & 60 & 3,740 & A & Ffo1 vs FMa+ & 0,905 \\
\hline Ffo1 & 60 & 3,840 & A & Ffo2 vs Ffo1 & 0,774 \\
\hline Ffo2 & 60 & 4,000 & A & Ffo2 vs FMa+ & 0,511 \\
\hline
\end{tabular}

Farine à base de maïs (FMa+) ; Farine à base de fonio 1 (Ffo1) ; Farine à base de fonio 2 (Ffo2).

\section{DISCUSSION}

La présente étude avait pour objectif de proposer une formule de bouillie à base de fonio enrichie en ressources alimentaires locales, de valeur nutritive et qualité sanitaire adéquates, et acceptable par les enfants en âge de complémentation alimentaire. Nous avons réussi à développer une formule (Ffo2) avec $\mathrm{du}$ fonio $(60 \%)$ enrichi en soja $(20 \%)$, à l'arachide (16\%), et aux fretins séchés $(1 \%)$, conforme aux recommandations pour la composition en macronutriments et les caractéristiques physico-chimiques. $\mathrm{La}$ bouillie préparée à partir de cette farine a été acceptée par les mères comme aliment de complément pour leurs enfants pour sa consistance. Cependant chez les enfants, une nette préférence pour l'une des bouillies n'a pas été observée.

Il est recommandé que les bouillies de complément soient faites à partir de ressources alimentaires locales et qu'elles soient composées d'au moins $30 \%$ de matières sèches avec des densités énergétiques proches ou supérieures à $120 \mathrm{kcal} / 100 \mathrm{ml}$, afin que la prise de deux bouillies par jour puisse couvrir les besoins énergétiques journaliers de l'enfant (CAC, 2013). L'équilibre nutritionnel des formules de farines testées (et celle de fonio retenue) a été réalisé en combinant différents procédés de transformation des ressources locales alimentaires utilisées, selon les recommandations de la Commission du Codex Alimentarius (CAC, 2013). Du fait de sa disponibilité et de son accessibilité facile, le soja a été choisi comme principale source de protéine, à l'instar de la plupart des farines infantiles produites et commercialisées au Bénin (Dagbadji, 2003 ; Nago, 2012). Son utilisation se justifie surtout par sa composition équilibrée en protéines de bonne valeur biologique contenant tous les acides aminés essentiels ainsi que des vitamines et des minéraux (Laurent, 2011). Néanmoins, le soja est caractérisé par une forte activité antinutritionnelle qui a été réduite par la torréfaction. L'addition de poissons fretins (1\%) à la formule se justifie par le fait que ces derniers constituent une source de protéine d'origine animale apportant une meilleure 
digestibilité et facilitant l'absorption des protéines d'origine végétale. La torréfaction des ingrédients a permis de réduire leur teneur en eau, favorisant l'augmentation de la teneur en matière sèches des formules testées (CAC, 2013).

Récemment, deux expériences positives de formulation de farine infantile ont été rapportées en Côte d'Ivoire avec le manioc et le soja (Dègnon et al., 2015), et au Bénin avec le maïs, le fonio, et le poisson fretin (Zannou-Tchoko et al., 2011). Cependant, ces études n'ont pas rapporté d'information sur la densité énergétique des formules de farine obtenues. Dans la présente étude, les densités énergétiques des trois formules de farines testées sont élevées (495 à 498,72 Kcal par rapport à la norme $(400 \mathrm{Kcal})$. Elles sont également supérieures à celles des farines infantiles produites et commercialisées au Bénin (367 à $486 \mathrm{kcal} / \mathrm{g}$ ) (Dagbadji, 2003 ; Nago, 2012). Ceci pourrait s'expliquer par l'incorporation de $16 \%$ d'arachide, oléagineuse très énergétique avec 595 $\mathrm{kcal} / 100 \mathrm{~g}$ de matières sèches (FAO et al., 2012). L'incorporation de $20 \%$ de soja aurait également contribué à la densité énergétique, ainsi qu'à la teneur assez élevée en protéines et lipides des farines comme montré dans les études antérieures (Zannou-Tchoko et al. 2011 ; Suri et al., 2014 ; Dègnon et al., 2015 ; Tshite et al., 2015). Pour réduire la consistance épaisse des bouilles, la technique du maltage a été appliquée aux bouillies pour conserver leur densité énergétique tout en assurant une réelle couverture des besoins au vu des nombreux avantages de son utilisation. L'incorporation de malt entraîne une augmentation de la vitesse d'écoulement associée avec une augmentation de la matière sèche et de la densité énergétique des bouillies (Kayode et al., 2007; Elenga, 2012). La quantité de malt de maïs adaptée à la formulation a été retenue au cours des essais en tenant compte de la consistance des bouillies de fonio.

En dehors de l'équilibre nutritionnel et de l'innocuité des farines infantiles, leur acceptabilité sensorielle a été également appréciée, étant une condition essentielle de la consommation des bouillies par les enfants. La bouillie obtenue à partir de la formule Ffo2 de farine fonio a été acceptée par les mères sur la base de sa consistance moins épaisse que les deux autres formules testées. Aucune autre différence n'a été notée par les mères entre les bouillies au niveau des autres paramètres d'évaluation sensorielle (goût et couleur). Cela justifie aussi probablement le fait que les enfants n'aient détecté aucune différence entre les trois formules testées.

L'une des limites de la formule de farine Ffo2, (ainsi que les deux autres formules testées) réside dans sa faible teneur en fer $(2 \mathrm{mg})$ par rapport aux recommandations. En projetant une biodisponibilité limitée par l'acide phytique qui se trouve dans la plupart des aliments d'origine végétale (Zimmermann et Hurrell, 2007), il sera nécessaire d'améliorer la teneur en fer à travers la fortification à domicile. Il a été montré que la réduction quasi-totale des phytates et une supplémentation légère en fer permettait d'améliorer la biodisponibilité du fer dans les bouillies de fonio consommée par les femmes en âge de procréer (KoreissiDembélé et al., 2013). Cette technique pourra être adaptée pour les formules de farines de complément. Par ailleurs, la promotion de cette formule de farine à base de fonio pourrait être limitée par l'accessibilité géographique du fonio, céréale dont la production est concentrée dans la partie NordOuest du Bénin (Fogny-Fanou et al., 2009). Une option serait d'adapter cette formule aux ressources alimentaires locales disponibles selon les zones agroécologiques du Bénin. 


\section{Conclusion}

L'étude a permis de formuler et de développer une farine de fonio enrichie en ressources alimentaires locales, et répondant aux recommandations de l'OMS en termes de composition proximale pour l'alimentation complémentaire. Cette farine a été bien appréciée par les mères d'enfants de 6 à 59 mois. Aussi contribue-t-elle, en termes de qualité, à la diversité alimentaire minimale préconisée pour les enfants de 6 à 23 mois (WHO, 2008), en combinant au moins quatre groupes d'aliments sur les sept recommandés : les céréales (fonio), les légumineuses (soja), les graines (arachide), et les petits poissons séchés. La formule devrait être adaptée aux différentes zones agroécologiques du Bénin, et améliorée en micronutriments tels que fer, en exploitant les ressources alimentaires locales disponibles dans chaque zone.

\section{CONFLITS D'INTERETS}

Les auteurs déclarent n'avoir aucun conflit d'intérêts.

\section{CONTRIBUTIONS DES AUTEURS}

NF-F est le principal investigateur de la recherche. EMYM a participé à la conception méthodologique et a relu les différentes versions du manuscrit. YA-L a élaboré le protocole, collecté les données et rédigé le premier draft du manuscrit. FTFL a amélioré les versions du manuscrit. APPK a monitoré le groupe pour toute la recherche. Tous les auteurs ont lu et approuvé la version finale du manuscrit.

\section{REMERCIEMENTS}

Les remerciements vont aussi à l'endroit des assistants Lidwine Gnamlin et Augustin Akodjènou qui ont participé à la collecte de données, des animateurs de l'épicentre de Wawata de l'ONG The Hunger Project pour la mobilisation des ménages enquêtés, de l'Atelier 1000-et-une bouillies qui a aidé à préparer les bouillies pour le test d'évaluation sensorielle, et des mères ayant participé au test des formules de farines.

\section{REFERENCES}

AOAC (Association of Official Analytical Chemists). 2005. Official Methods of Analysis of the Association of Analytical Chemists International (18 $8^{\text {th }}$ edn). AOAC: Gathersburg, MD U.S.A.

Arimond M, Daelmans B, Dewey KG. 2008. Indicators for Feeding Practices in Children. The Lancet, 371: 541-542.

Black RE, Makrides M, Ong KK. 2017. Complementary Feeding: Building the Foundations of Healthy Life. Nestlé Nutrition Institute Workshops Series (vol 87). Basel, Switzerland, Karger; Vevey, Nestlé: Switzerland.

Brou K, Kouadio EJPN, Due EA, Kouame SK, Tano K, Dago G. 2009. Effects of processing method and blend on some physicochemical properties and digestibility of flours made from selected cereals and legumes. Int. J. Biol. Chem. Sci., 3(5): 1151-1160.

CAC (Commission du Codex Alimentarius). 2013. Lignes directrices pour la mise au point des préparations alimentaires complémentaires destinées aux nourrissons du deuxième âge et aux enfants en bas âge CAC/GL 08-1991. Adoptée en 1991; Révisée en 2013. Programme mixte FAO/OMS sur les normes alimentaires, Comité du Codex sur la nutrition et les aliments diététiques ou de régime. www.fao.org/input/download/standards/ 298/CXG_008e.pdf [Accédé le 11 mai 2017].

Dagbadji RR. 2003. Evaluation de la qualité de quelques aliments de complément commercialisés au Benin. Thèse d'Ingénieur. Agronome, FSA / UAC, p90. 
Dègnon GR, Adjou ES, Challa CC, Acodehou CS. 2015. Investigation on the quality of some traditional infant flour formulations used for the nutritional recovery of children in Benin. Int. $J$. Pharm. Sci. Rev. Res., 31(2): 259-264.

Elenga M, Massamba J, Kobawila SC, Makosso VG, Silou T. 2009. Evaluation et amélioration de la qualité nutritionnelle des pâtes et des bouillies de maïs fermenté au Congo. Int. J. Biol. Chem. Sci., 3(6): 1274-1285.

Elenga M. 2012. Effet de l'incorporation de malt sur la fluidité et la densité énergétique des bouillies de maïs arachide destinées aux nourrissons et aux jeunes enfants. J Applied Biosciences, 55: 3995 - 4005.

FAO (Food and Agriculture Organisation) / WHO (World Health Organisation) 1998. Carbohydrates in human nutrition. Report of a joint FAO/WHO expert consultation. FAO Food and Nutrition Paper: Rome.

FAO (Food and Agriculture Organisation), INFOODS (International Network of Food Data Systems), WAHO (West African Health Organisation), Bioversity International. 2012. West African Food Composition Table. 978-92-5-007207-4. Rome; www.fao.org/docrep/015/i2698b/ i2698b00.pdf [Accédé le 11 mai 2017].

Fogny-Fanou N, Koreissi Y, Dossa RAM, and ID Brouwer. 2009. Consumption of, and beliefs about fonio (Digitaria exilis) in urban area in Mali. African Journal of Food Agriculture Nutrition and Development, 9(9): 1927 - 1944.

IFPRI (International Food Policy Research Institute), 2014. Global Nutrition Report 2014: Actions and Accountability to Accelerate the World's Progress on Nutrition: Washington, DC.

INSAE (Institut National de la Statistique et de l'Analyse Économique) et ICF
International. 2013. Enquête Démographique et de Santé du Bénin 2011-2012. INSAE et ICF International : Calverton, Maryland, USA.

Kayode AP, Hounhouigan JD, Nout MJ. 2007 Impact of brewing process operation on phytate, phenolic compounds and in vitro solubility of iron and zinc in opaque sorghum beer. $L W T, 40: 834-$ 841.

Kimiywe J, Chege PM. 2015. Complementary feeding practices and nutritional status of children 6-23 months in Kitui County, Kenya. J Applied Biosciences, 85: 78817890.

Koreissi-Dembélé Y, Fanou-Fogny N, Hulshof PJM, Brouwer ID. 2013. Fonio (Digitaria exilis) landraces in Mali: Nutrient and phytate content, genetic diversity and effect of processing. Journal of Food Composition and Analysis, 29: 134-143.

Kumari S, Ichhpujani RL. 2000. Guidelines on Standard Operating Procedures for Microbiology. WHO : Geneva.

Laurent X. 2011. Le soja, Pourquoi ? Comment ? Formation BAMiSA, Thiès, Sénégal.

www.bamisagora.org/documents_pdf/do cument11a-bamisagora.pdf [Accédé 17 janvier 2017].

Mouquet C, Salvignol B, Van Hoan N, Monvois J, Trèche S. 2003. Ability of a very low cost extruder to produce instant infant flours at a small scale in Vietnam. Food Chemistry, 82: 249-255.

Mouquet C, Trèche S. 2001. Viscosity of gruels for infants a comparison of measurement procedures. Int. J. Food Sci. Nut., 52: 389-400.

Nago MIS. 2012. Evaluation de la qualité nutritionnelle des farines infantiles fabriquées et vendues au Bénin. Thèse de Docteur en pharmacie. Unité de formation et de recherche en pharmacie, 
Faculté des Sciences de la Santé, Université d'Abomey-Calavi, Bénin.

OMS (Organisation Mondiale de la Santé), 2015. Alimentation du nourrisson et du jeune enfant. Aide-mémoire $\quad N^{\circ} 342$, Juillet 2015 http://www.who.int/mediacentre/factshee ts/fs342/fr/ [Accédé le 11 mai 2017].

Osborne DR, Voogt P. 1978. The Analysis of Nutrients in Foods. Food Science and Technology: A Series of Monographs. Academic Press: London.

Popper R, Kroll JJ. 2005. Conducting sensory research with children. J. Sensory Studies, 20: 75-87.

Suri DJ, Tano-Debrah K, Ghosh SA. 2014. Optimization of the nutrient content and protein quality of cereal - legume blends for use as complementary foods in Ghana. Food and Nutrition Bulletin, 35(3): $372-381$.

Toledo A, Burlingame B. 2006. Biodiversity and nutrition: A common path toward global food security and sustainable development. Journal of Food Composition and Analysis, 19: 477-483.

Trêche S. 1999. Logiciel d'aide à la formulation d'aliments composés. Laboratoire de Nutrition Tropicale de l'ORSTOM (LNT). Bulletin du Réseau TPA: $\mathrm{N}^{\circ} 15$. France.
Tshite FN, Mulamba VT, Ndianabo MJT. 2015. Mise au point d'une farine précuite à base de maïs (Zea mays) et de soja (Glycine max) par la méthode traditionnelle. Int. J. Biol. Chem. Sci., 9(6): 2608-2622. DOI: http://dx.doi.org/10.4314/ijbcs.v9i6.8.

Vaahtera M, Kulmala T, Hietanen A, Ndekha M, Cullinan T, Salin M-L, Ashorn P. 2001. Breastfeeding and complementary feeding practices in rural Malawi. Acta Pcediatr., 90: 328-332.

WHO (World Health Organization). 2008. Indicators for Assessing Infant and Young Child Feeding Practices. Conclusions of a Consensus Meeting held 6-8 November 2007 in Washington, DC, USA. WHO: Geneva; available at http://whqlibdoc.who.int/publications/20 08/9789241596664_eng.pdf

Zannou-Tchoko VJ, Bouaffou KGM, Kouame KG, Konan BA. 2011. Etude de la valeur nutritive de farines infantiles à base de manioc et de soja pour enfant en âge de sevrage. Bull Soc Royale Sci Liège, 80: $748-758$.

Zimmermann MB, Hurrell RF. 2007. Nutritional iron deficiency. The Lancet, 370 (9586): 511-520. 